\title{
Comparison of target volume and clinical effects of four radiotherapy plans for acute lymphoblastic leukemia prior to hematopoietic stem cell transplantation
}

\author{
YALEI LIN*, FANYANG KONG* , HONGFEI LI, DANDAN XU, FEI JIA, \\ XUDONG ZHANG, BAOHONG WANG and GUOWEN LI \\ Department of Radiation Oncology, The First Affiliated Hospital of Zhengzhou University, \\ Zhengzhou, Henan 450052, P.R. China
}

Received October 16, 2017; Accepted April 13, 2018

DOI: $10.3892 / \mathrm{mmr} .2018 .9228$

\begin{abstract}
The present study aimed to investigate the variations in target volume, clinical reaction and transplantation effects of helical tomotherapy (HT)-total body irradiation (TBI), HT-total marrow and lymphatic irradiation (TMLI), intensity modulated radiotherapy (IMRT)-TBI and IMRT-TMLI within patients with acute lymphoblastic leukemia (ALL). A total of 18 patients with ALL were treated with the four aforementioned radiotherapy plans prior to hematopoietic stem cell transplantation. A planned prescribed dose of 12 Gy/6 Frequency was administered to determine planning target volume (PTV). Dosimetry evaluation indexes in PTV and organs at risk were analyzed. Comparison of clinical untoward effects and the results of transplantation among the four plans were performed. The conformity index of HT plans was significantly increased compared with those in IMRT plans. The mean dose (D) to the lung and volume ratio of target volume occupied by $5 \mathrm{~Gy}\left(\mathrm{~V}_{5}\right)$ in TMLI plans were lower compared with TBI plans. Doses to organs were controlled within the normal range. $\mathrm{D}_{\text {max }}, \mathrm{D}_{\text {mean }}$ and $\mathrm{V}_{5}$ of bilateral lungs and $\mathrm{D}_{\max }$ and $\mathrm{D}_{\text {mean }}$ of bilateral crystalline lens in IMRT plans were significantly higher compared with HT plans. There were no significant differences in untoward effects among the four plans. Subsequent to symptomatic treatments with antiemetic, antidiarrheal and fluid infusion, untoward effects improved, and all patients demonstrated tolerance to these therapies. A total of six patients treated with HT-TBI revealed
\end{abstract}

Correspondence to: Dr Guowen Li, Department of Radiation Oncology, The First Affiliated Hospital of Zhengzhou University, 1 Eastern Jianshe Road, Zhengzhou, Henan 450052, P.R. China

E-mail: guowenli12@sohu.com

${ }^{*}$ Contributed equally

Key words: acute lymphoblastic leukemia, hematopoietic stem cell transplantation, radiotherapy, total body irradiation, total marrow and lymphatic irradiation, dosimetry complete and successful transplantation; however, one patient following transplantation suffered from severe rejection and had succumbed to mortality due to severe infection. Patients treated with HT-TMLI, IMRT-TBI and IMRT-TMLI completed successful transplantation and no rejection responses were observed. Conformity of HT plans are higher than that of IMRT plans. The four radiotherapy plans exhibit similar clinical untoward effects and the same transplantation success rate. HT-TMLI is more feasible in dosimetry compared with HT-TBI, IMRT-TBI and IMRT-TMLI, which require further long-term observation.

\section{Introduction}

Acute lymphoblastic leukemia (ALL), with a high invasion, is a malignant heterogeneous disease occurring in lymphoid hematopoietic cells (1). The etiology and pathogenesis of ALL remain to be investigated. There is a great heterogeneity between the treatment and the prognosis of ALL. ALL often occurs in children, accounting for $70-80 \%$ of children with acute leukemia (2). Patients with ALL usually have a poor prognosis due to variable pathogenesis, complex etiology, severe clinical symptoms and complex treatment plans, which may place a burden on children's physical and mental health. With the progression of clinical treatment methods, the therapeutic effect of ALL treatment has increased. For children, the five-year overall survival rate has reached $92.3 \%$, while the rate was only $\sim 10 \%$ in the 1960 s; the five-year disease-free survival (DFS) rate has also increased to 80-85\%. In addition, the total remission rate of simple chemotherapy in children is $>90 \%$, and the DFS is $\leq 70-80 \%(3,4)$. In the 1990 s, the five-year survival rate of children with ALL in developed countries was reported to be $70-80 \%$, and the overall cure rate reached $80 \%$. The cure rate of children with leukemia is predicted to be $>90 \%$ in China in the near future with the development of medical technology (5). The incidence rate of ALL in adults is lower compared with children, but adults with ALL exhibit poor effects of treatment, $\leq 40 \%$ of relapse rate and only $30-40 \%$ DFS (6). For adults, conventional chemotherapy serves a poor effect; $70-80 \%$ patients may not survive for a long period or be cured, requiring long-term treatment (7). 
The primary clinical treatment method for ALL is combination chemotherapy. Targeted therapy and hematopoietic stem cell transplantation are also methods of treatment. In addition to chemotherapy, hematopoietic stem cell transplantation is another important method of treatment for patients with ALL. Simple high-dose chemotherapy is not able to serve a myeloablative effect. Due to the limitations of chemotherapeutic drugs, it is difficult to eliminate tumor cells detected in sheltered areas by chemotherapy, leading to tumor cell recurrence (6). Chemotherapy combined with radiotherapy has become an important method of pretreatment prior to hematopoietic stem cell transplantation with developing radiotherapy technology. It is clinically confirmed that the application of total body irradiation (TBI) may improve the success rate of hematopoietic stem cell transplantation and decrease the rate of recurrence (8).

TBI is an important method of pretreatment prior to hematopoietic stem cell transplantation $(9,10)$. The effective pretreatment method of hematopoietic stem cell transplantation is large-dose chemotherapy combined with TBI, which prompts successful transplantation (11). The Northern European Bone Marrow Transplant Organization, via relevant clinical trials, have demonstrated that chemotherapy combined with TBI may exhibit an increased effect of pretreatment compared with simple chemotherapy for children or adults with acute leukemia, and that chemotherapy combined with TBI may provide improved survival conditions for patients (12-15). The three-year overall survival rate of patients treated with chemotherapy combined with TBI following transplantation is $76 \%$, which is higher than $62 \%$ simple chemotherapy, and the patients have a long DFS and a low recurrence rate (12-15). As of the 20th century, chemotherapy combined with TBI has demonstrated improved effects for children or adults with leukemia compared with simple chemotherapy (16-18). Linsenmeier et al (19) indicated that TBI was a safe and effective pretreatment method prior to hematopoietic stem cell transplantation; TBI prior to high-dose chemotherapy may maintain the same effects of pretreatment and effectively reduce notable untoward effects or discomfort, the preparation workload of radiotherapy and the untoward effects of radiation (19). Bhatia et al $(20,21)$ revealed that TBI had a preventive effect on the late mortality for transplantation patients and that patients treated with TBI had lower rates of late mortality by $\geq 50 \%$ compared with patients without TBI.

However, with the increase and development of clinical application, limitations of TBI have been reported. Clinical experience has demonstrated that it is inadvisable to rely solely on increasing doses of TBI, which can lead to an increase of the dose to organs at risk including the lungs, livers and kidneys, enhancing the incidence of complications, and an unimproved overall survival rate for patients with ALL, therefore, a more targeted TBI should be developed to lessen toxic reactions while increasing doses (22). The application of total marrow and lymphatic irradiation (TMLI) to the whole-body skeleton, main lymph node chain and the spleen may evoke a sufficient immunosuppression response to the transplant (23). Studies have revealed that patients treated with TMLI and TBI have an equivalent extramedullary relapse rate following hematopoietic stem cell transplantation; however, TMLI is a more conservative targeted radiotherapy and does not increase the risk of extramedullary relapse (23-27). TMLI may reduce toxic reactions while increasing doses to target volumes, which provide the possibility of treatment to the elderly and patients with complications who poorly suit to conventional TBI $(28,29)$. Tomotherapy accelerators have gradually replaced the intensity-modulated radiotherapy (IMRT) of the conventional accelerator.

By comparing the target volume, clinical reaction and effects of transplantation among helical tomotherapy (HT)-TBI, HT-TMLI, IMRT-TBI and IMRT-TMLI, the present study evaluated the advantages and disadvantages of these methods of treatment and analyzed the possibility of replacing TBI with TMLI in clinical treatment, which may provide a theoretical basis to clinical treatment.

\section{Materials and methods}

Patients. A total of 18 patients with ALL treated with total body irradiation prior to hematopoietic stem cell transplantation in the First Affiliated Hospital of Zhengzhou University (Zhengzhou, China) from May 2016 to December 2016 were selected. In the present study, 10 males and eight females, with an age range of 4-34 years old were enrolled. The Karnofsky performance status was $>100$ (30). According to pathological anatomy of the 18 patients, there were nine patients with T-cell acute lymphoblastic leukemia and nine patients with B-cell acute lymphoblastic leukemia. Prior to radiotherapy, all patients and their families were notified of the requirement of treatment and possible untoward effects, and signed written informed consent was obtained. All patients prior to radiotherapy underwent systemic high-dose chemotherapy plus intrathecal injection. Following radiotherapy, patients entered into the isolation room on the same day; after 1- to 2-day preparation, myeloablative transplantation therapy was performed. The inclusion criteria were as follows: i) Definite pathological diagnosis; ii) systemic chemotherapy plus intrathecal injection; and iii) normal heart, liver and kidney function and Karnofsky performance status $\geq 80$. The exclusion criteria were as follows: i) severe damage of heart, liver, kidney and lung function; ii) severe or uncontrolled infection; and iii) patients with a history of severe mental disorder that are not compliant to treatment. The present study was approved by the Ethics Committee of the First Affiliated Hospital of Zhengzhou University.

Computed tomography (CT) localization, scan and image registration. A thermoplastic mask and vacuum pad were provided to patients, according to the CT localization requirement of radiotherapy, and the lead points were affixed at three points. For patients with the height of $>120 \mathrm{~cm}$, there were three lead points, each on two sets of images, respectively located $5-10 \mathrm{~cm}$ above the navel and $5-10 \mathrm{~cm}$ below the knee. A continuous flat scan was performed with Definition AS large aperture CT analog positioning machine (Siemens AG, Munich, Germany). CT images of the phase during normal respiration were acquired. As the maximum length of the tomotherapy machine was $160 \mathrm{~cm}$, a segmented scan was performed for patients with a height $>120 \mathrm{~cm}$.

Delineating the clinical target volume (CTV) in CT location images (segmented delineation for patients with a height 


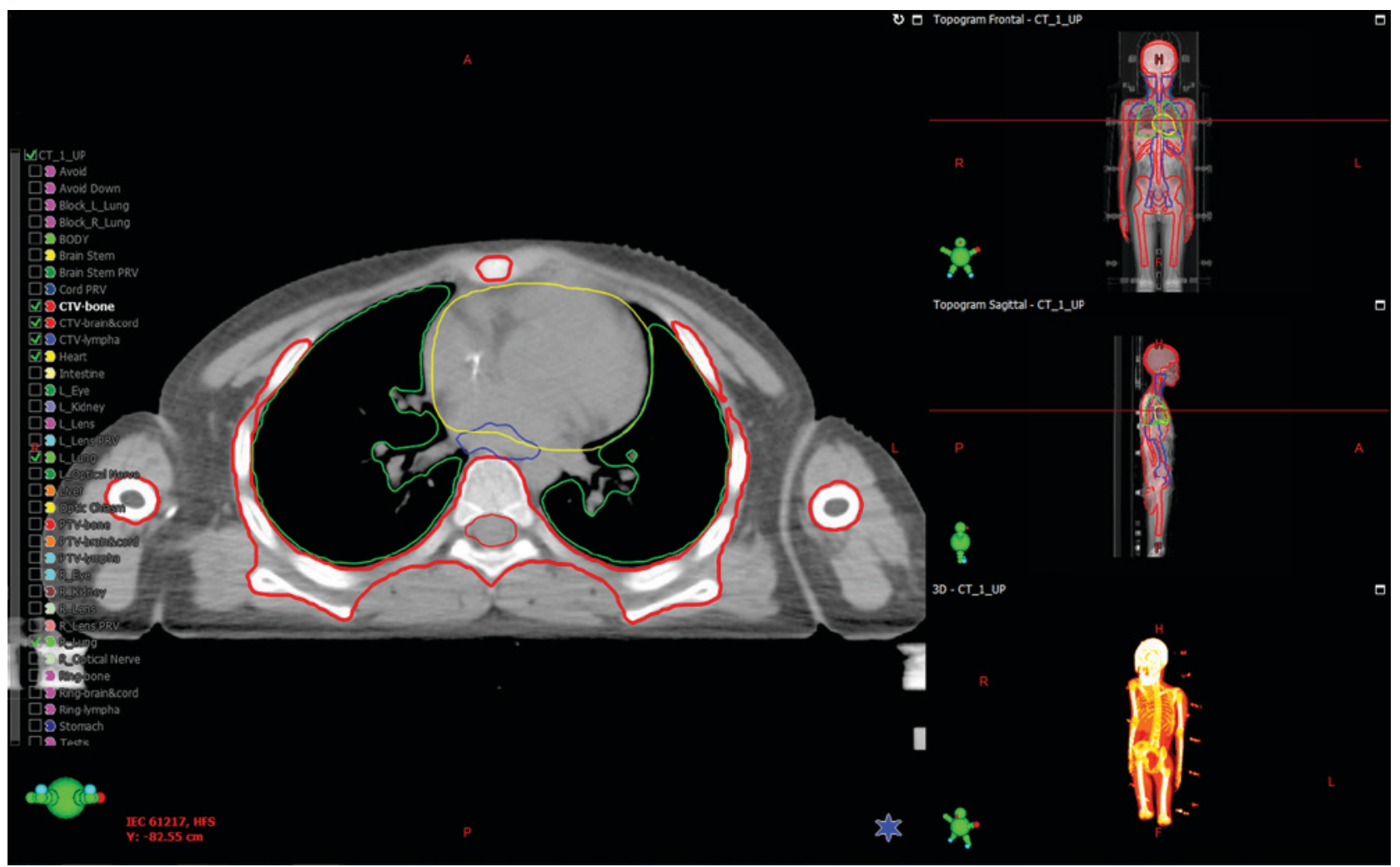

Figure 1. Target volume delineation and organs at risk. Clinical target volume is outlined in red; the green line area demonstrated double lungs and the yellow line area presented the spinal cord and the heart.

$>120 \mathrm{~cm})$. CTV of TBI constituting the whole body except the organs at risk, usually with $3 \mathrm{~mm}$ of skin adduction as CTV; CTV of TMLI constituted the whole body bone marrow (sternum, vertebra, rib, to delineate conveniently target volume), main lymph node chains (neck and mediastinal lymph nodes) and the spleen. Considering the reproduction of patients, and the intentions and chemotherapy plans of the patient and their families in the clinical treatment, the testes were not irradiated. Planning target volume (PTV) was delineated by externally extending $5 \mathrm{~mm}$ from every direction of CTV. Organs at risk included bilateral crystalline lens and bilateral lungs (Fig. 1).

Prescription dose setting for target volume, organs at risk and $P T V .2$ Gy were administered each time, two times a day, with an interval of 6-8 h and 6 total doses of $12 \mathrm{~Gy}$.

Dose constraint for important organs at risk. According to the requirement of the United States Radiation Therapy Oncology Group (31); dose constraint in the main normal tissues is presented in Table I.

Radiotherapy planning. CT images of the target volume and organs at risk were transferred to the Tomotherapy and Pinnacle V9.8.6008 treatment planning system and the same physicist conducted the radiotherapy plans according to the requirements of the clinician. IMRT of the conventional accelerator was conducted using a Primus linear accelerator with 6 megavoltage (MV) X rays and 3-7 radiation fields. HT was implemented with $6 \mathrm{MV}$ X rays; most lung tissues on one side or on dorsal and ventral sides avoided exposure to radiation.
Patients 1-10 were treated with HT plans and assigned to groups randomly; patients 1-6 target volume delineation was via HT-TBI, and patients 7-10 via HT-TMLI. Patients 11-18 were treated with IMRT plans; patients 11-14 target volume delineation was via IMRT-TBI, and patients $15-18$ via IMRT-TMLI. The associated indexes of target volume and organs at risk in different radiotherapy plans were recorded and compared.

Dosimetry evaluation indexes. According to the dose volume histogram (DVH) of each plan, the dosimetry evaluation indexes in target volume and organs at risk included: $\mathrm{D}_{\max }$, maximum dose to target volume or organs at risk (cGy); $\mathrm{D}_{\text {mean }}$, average dose to target volume or organs at risk (cGy); $\mathrm{D}_{98}$, dose to $98 \%$ of target volume or organs at risk (cGy); $\mathrm{D}_{95}$, dose to $95 \%$ of target volume or organs at risk (cGy); $\mathrm{D}_{50}$, dose to $50 \%$ of target volume or organs at risk (cGy); $\mathrm{D}_{02}$, dose to $2 \%$ of target volume or organs at risk (cGy) and $\mathrm{V}_{12}$, volume ratio of target volume occupied by $12 \mathrm{~Gy}$.

Dose to double lungs prior to hematopoietic stem cell transplantation revealed close associations with the complications following transplantation. Evaluation indexes for double lungs included $\mathrm{D}_{\max }$ and $\mathrm{D}_{\text {mean }}$; however, considering the specificity of TBI in target volume, the average dose to the lung should be lower than $8 \mathrm{~Gy}$ when evaluating the dose to the lung during the treatment.

Evaluation indexes for the crystalline lens included $\mathrm{D}_{\max }$ and $\mathrm{D}_{\text {mean }}$. In addition, conformity index (CI) and homogeneity index (HI) of target volume in HI and IMRT plans were compared.

Clinical untoward effects and transplantation effects. The main untoward effects occurred during the treatment, 
Table I. Radiotherapy dose constraint in the normal tissues.

\begin{tabular}{llc}
\hline Organ at risk & Dose constraint & \multicolumn{1}{c}{ PRV } \\
\hline Lung & Mean dose $<8$ Gy & \\
Left Lens & Max $\leq 9$ Gy & PRV external expansion \\
& & 3 mm, Max $<9$ Gy \\
Right Lens & Max $\leq 9$ Gy & PRV external expansion \\
& & $3 \mathrm{~mm}$, Max $<9$ Gy \\
\hline
\end{tabular}

PRV, planning risk volume.

Table II. Basic data of patients.

\begin{tabular}{llrll}
\hline $\begin{array}{l}\text { Patient } \\
\text { number }\end{array}$ & Sex & Age & $\begin{array}{c}\text { Pathological } \\
\text { type }\end{array}$ & Complications \\
\hline 1 & Female & 7 & B-cell ALL & No \\
2 & Male & 7 & B-cell ALL & No \\
3 & Male & 13 & T-cell ALL & No \\
4 & Female & 12 & B-cell ALL & No \\
5 & Male & 10 & T-cell ALL & No \\
6 & Male & 9 & T-cell ALL & No \\
7 & Female & 19 & T-cell ALL & No \\
8 & Female & 34 & B-cell ALL & No \\
9 & Male & 12 & B-cell ALL & No \\
10 & Male & 6 & T-cell ALL & No \\
11 & Female & 8 & T-cell ALL & No \\
12 & Female & 13 & B-cell ALL & No \\
13 & Male & 16 & T-cell ALL & No \\
14 & Male & 21 & B-cell ALL & No \\
15 & Male & 18 & B-cell ALL & No \\
16 & Female & 10 & T-cell ALL & No \\
17 & Male & 11 & B-cell ALL & No \\
18 & Female & 7 & T-cell ALL & No \\
\hline
\end{tabular}

ALL, acute lymphoblastic leukemia.

including nausea, emesis, inappetence, xerostomia, fever, diarrhea and rash, among of which nausea, emesis, inappetence and xerostomia were the most common reactions. All reactions were at I-II level (32). Symptomatic treatment was performed, according to the patients' specific condition. Patients suffering from fever, received treatment; physical cooling for patients with low fever and aspirin-DL-lysine for patients with a body temperature $>38.5^{\circ} \mathrm{C}$.

Transplantation effects were assessed by whether the 18 patients had successful transplantation or not. The number of survivors and those who had succumbed to mortality, and the cause of mortality during long-term follow-up was recorded.

Statistical analysis. The statistical software SPSS (version 17.0; SPSS, Inc., Chicago, IL, USA) was employed to analyze the data in the present study. All data were presented as the mean \pm standard deviation. Provided that the difference
Table III. The dose distribution in PTV between HT and IMRT plans (cGy).

\begin{tabular}{lcccc}
\hline A, TBI & \multicolumn{5}{c}{} \\
\hline Variable & HT & IMRT & t-test & P-value \\
\hline $\mathrm{D}_{\max }$ & $12.250 \pm 0.606$ & $12.050 \pm 0.592$ & 0.35 & 0.75 \\
$\mathrm{D}_{98}$ & $11.128 \pm 0.176$ & $11.124 \pm 0.181$ & 0.08 & 0.95 \\
$\mathrm{D}_{95}$ & $11.940 \pm 0.689$ & $11.923 \pm 0.653$ & 0.32 & 0.73 \\
$\mathrm{D}_{\text {mean }}$ & $12.453 \pm 0.092$ & $12.782 \pm 0.085$ & -0.32 & 0.77 \\
$\mathrm{D}_{50}$ & $12.527 \pm 0.124$ & $12.432 \pm 0.119$ & 0.05 & 0.96 \\
$\mathrm{D}_{02}$ & $11.370 \pm 4.167$ & $11.115 \pm 4.059$ & 0.33 & 0.72 \\
$\mathrm{~V}_{12}$ & $94.541 \pm 0.979$ & $92.521 \pm 0.955$ & 0.98 & 0.338 \\
$\mathrm{HI}$ & $1.100 \pm 0.050$ & $1.110 \pm 0.060$ & -0.78 & 0.423 \\
$\mathrm{CI}$ & $0.770 \pm 0.030$ & $0.710 \pm 0.025$ & 4.41 & $<0.001$ \\
\hline
\end{tabular}

B, TMLI

\begin{tabular}{lcccc}
\hline Variable & HT & IMRT & t-test & P-value \\
\hline $\mathrm{D}_{\max }$ & $13.930 \pm 0.336$ & $13.890 \pm 0.351$ & 0.33 & 0.88 \\
$\mathrm{D}_{98}$ & $10.931 \pm 0.452$ & $11.124 \pm 0.449$ & -0.32 & 0.74 \\
$\mathrm{D}_{95}$ & $11.922 \pm 0.174$ & $11.920 \pm 0.158$ & 0.88 & 0.92 \\
$\mathrm{D}_{\text {mean }}$ & $12.720 \pm 0.202$ & $12.698 \pm 0.211$ & 0.35 & 0.85 \\
$\mathrm{D}_{50}$ & $12.910 \pm 0.297$ & $12.831 \pm 0.287$ & 0.75 & 0.59 \\
$\mathrm{D}_{02}$ & $13.283 \pm 0.095$ & $12.950 \pm 0.096$ & 0.36 & 0.68 \\
$\mathrm{~V}_{12}$ & $94.360 \pm 0.961$ & $93.537 \pm 0.954$ & 0.41 & 0.78 \\
$\mathrm{HI}$ & $1.120 \pm 0.040$ & $1.110 \pm 0.030$ & 0.75 & 0.42 \\
$\mathrm{CI}$ & $0.810 \pm 0.030$ & $0.770 \pm 0.050$ & 4.56 & $<0.001$ \\
\hline
\end{tabular}

TBI, total body irradiation; TMLI, total marrow and lymphatic irradiation; HT, helical tomotherapy; IMRT, intensity modulated radiotherapy; HI, homogeneity index; CI, conformal index; D, dose.

between the groups followed the normal distribution, the data were analyzed by a Student's t-test. If the difference between groups did not follow the normal distribution, the data were analyzed by a Wilcoxon rank-sum test. Multiple comparisons were analyzed by using one-way analysis of variance with the post hoc Student-Newman-Keuls test. $\mathrm{P}<0.05$ was considered to indicate a significant difference.

\section{Results}

Radiotherapy plans comparison. Basic clinical data of the 18 patients were presented in Table II. Patients 1-6 target volume delineation was according to HT-TBI and patients 7-10 via HT-TMLI. Patients 11-14 target volume delineation was according to IMRT-TBI and patients 15-18 via IMRT-TMLI.

Dose distribution in the target volume of HT and IMRT radiotherapy plans met dose requirements. CI in HT plans was significantly higher compared with IMRT plans $(\mathrm{P}<0.05)$. Additionally, there was no significant difference in other indexes $(\mathrm{P}>0.05)$. The target volume of HT plans revealed a more homogeneous dose distribution and increased conformity compared with IMRT plans with the conventional accelerator. 
Table IV. Differences of doses in organs at risk between TBI plans and TMLI plans (cGy).

\begin{tabular}{|c|c|c|c|c|}
\hline \multirow[b]{2}{*}{ Variable } & \multicolumn{2}{|c|}{ HT } & \multicolumn{2}{|c|}{ IMRT } \\
\hline & TBI & TMLI & TBI & TMLI \\
\hline \multicolumn{5}{|l|}{ Left lung } \\
\hline $\mathrm{D}_{\max }$ & $12.208 \pm 1.835$ & $12.335 \pm 0.385$ & $13.587 \pm 1.796^{\mathrm{a}}$ & $13.956 \pm 0.652^{\mathrm{b}}$ \\
\hline $\mathrm{D}_{\text {mean }}$ & $7.637 \pm 0.400$ & $7.243 \pm 0.290^{c}$ & $8.356 \pm 0.389^{\mathrm{a}}$ & $7.961 \pm 0.286^{\mathrm{b}}$ \\
\hline $\mathrm{V}_{5}$ & $84.486 \pm 5.377$ & $80.417 \pm 2.980$ & $88.354 \pm 5.255^{\mathrm{a}}$ & $83.441 \pm 2.756^{\mathrm{b}}$ \\
\hline \multicolumn{5}{|c|}{ Right lung } \\
\hline $\mathrm{D}_{\max }$ & $12.045 \pm 1.665$ & $13.023 \pm 0.462$ & $12.958 \pm 1.586^{\mathrm{a}}$ & $13.156 \pm 0.432^{\mathrm{b}}$ \\
\hline $\mathrm{D}_{\text {mean }}$ & $7.540 \pm 0.491$ & $6.960 \pm 0.420^{c}$ & $8.012 \pm 0.512^{\mathrm{a}}$ & $7.223 \pm 0.410^{b}$ \\
\hline $\mathrm{V}_{5}$ & $82.392 \pm 2.565$ & $75.710 \pm 2.262^{c}$ & $87.256 \pm 2.554^{\mathrm{a}}$ & $80.004 \pm 2.353^{b}$ \\
\hline \multicolumn{5}{|l|}{ Left lens } \\
\hline $\mathrm{D}_{\max }$ & $2.002 \pm 0.573$ & $1.856 \pm 0.301$ & $2.520 \pm 0.551^{\mathrm{a}}$ & $1.958 \pm 0.334^{\mathrm{b}}$ \\
\hline $\mathrm{D}_{\text {mean }}$ & $1.678 \pm 0.221$ & $1.670 \pm 0.240$ & $1.785 \pm 0.201^{\mathrm{a}}$ & $1.754 \pm 0.231^{\mathrm{b}}$ \\
\hline \multicolumn{5}{|c|}{ Right lens } \\
\hline $\mathrm{D}_{\max }$ & $2.012 \pm 0.564$ & $1.900 \pm 0.138$ & $2.112 \pm 0.560^{\mathrm{a}}$ & $1.956 \pm 0.135^{\mathrm{b}}$ \\
\hline$D_{\text {mean }}$ & $1.678 \pm 0.243$ & $1.713 \pm 0.210$ & $1.681 \pm 0.253^{\mathrm{a}}$ & $1.799 \pm 0.230^{b}$ \\
\hline
\end{tabular}

${ }^{a} \mathrm{P}<0.05$ vs. HT-TBI; ${ }^{\mathrm{b}} \mathrm{P}<0.05$ vs. HT-TMLI; ${ }^{\mathrm{C}} \mathrm{P}<0.05$ vs. HT-TBI. D, dose; HT, helical tomotherapy; IMRT, intensity modulated radiotherapy; TBI, total body irradiation; TMLI, total marrow and lymphatic irradiation.

As presented in Table III, $\mathrm{D}_{95}$ of PTV in each radiotherapy plan was approximately equal to the prescribed dose of $12 \mathrm{~Gy}$. The target volume of TBI was larger, which met the requirement of radiotherapy plans. There were no significant differences in $\mathrm{D}_{\max }, \mathrm{D}_{98}, \mathrm{D}_{02}$ and $\mathrm{D}_{\text {mean }}$ among the four radiotherapy plans $(\mathrm{P}>0.05)$.

Table IV presented the results of dosimetry comparison in bilateral lung and bilateral crystalline lens among four radiotherapy plans. The average dose to the lung in each plan may be controlled under $8 \mathrm{~Gy}$. TMLI plans reduced the average dose to both lung and the volume ratio of target volume occupied by $5 \mathrm{~Gy}\left(\mathrm{~V}_{5}\right)$ compared with TBI plans, and the other indexes were within the dose limitation of normal tissue, without significant differences. $D_{\text {max }}, D_{\text {mean }}$ and $V_{5}$ of bilateral lungs and $D_{\max }$ and $\mathrm{D}_{\text {mean }}$ of bilateral crystalline lens in IMRT plans were significantly increased compared with HT plans $(\mathrm{P}<0.05)$.

By comparing the dose to PTV and organs at risk among the four different radiotherapy plans, all plans were able to meet dosimetry requirements in target volume and organs at risk. In addition, the dose to bilateral lung and bilateral crystalline lens in HT-TMLI was decreased compared with other plans. HT-TMLI exhibited less damage to the bilateral lung and bilateral crystalline lens.

Comparison of clinical untoward effects. Each patient prior to treatment was administered anti-emetic and antipyretic drugs to prevent emesis and fever. The untoward effects of 18 patients during treatment were recorded. Symptomatic treatment was performed, according to patients' specific situation.

Nausea and emesis. Nausea and emesis was observed in 18 patients. Emesis occurred following the second treatment, with I-II level. Emesis was improved following the administration of anti-emetic drugs, which did not affect the implementation of radiotherapy plan.

Inappetence. There was mild inappetence in 18 patients. Inappetence occurred following the third treatment. Inappetence improved following fluid replacement therapy, which did not affect the implementation of the radiotherapy plan.

Fever. There was fever in four patients (two treated with IMRT-TBI, one with IMRT-TMLI and one with HT-TMLI) at body temperature of $37.9-38.2^{\circ} \mathrm{C}$ in the first and second treatment. Fever had improved following the treatment with physical cooling at low fever or aspirin-DL-lysine at body temperature $>38.5^{\circ} \mathrm{C}$, which did not affect the implementation of radiotherapy plan.

Xerostomia. There was slight to moderate xerostomia in 18 patients, and red and swollen oral mucosa in six patients. Xerostomia occurred following the second treatment. Symptomatic treatment was performed and patients were instructed to drink more water and to maintain oral hygiene. All patients demonstrated tolerance to xerostomia, which did not affect the implementation of radiotherapy plan.

Diarrhea. Mild diarrhea was reported in five patients (two treated with IMRT-TBI, one with IMRT-TMLI, one with HT-TBI and one with HT-TMLI) following the treatment. Diarrhea was improved following the administration of antidiarrheal drugs, which did not affect the implementation of radiotherapy plan.

Rash. Mild rashes were recorded in two patients treated with TBI (one with HT-TBI and one with IMRT-TBI) following the 


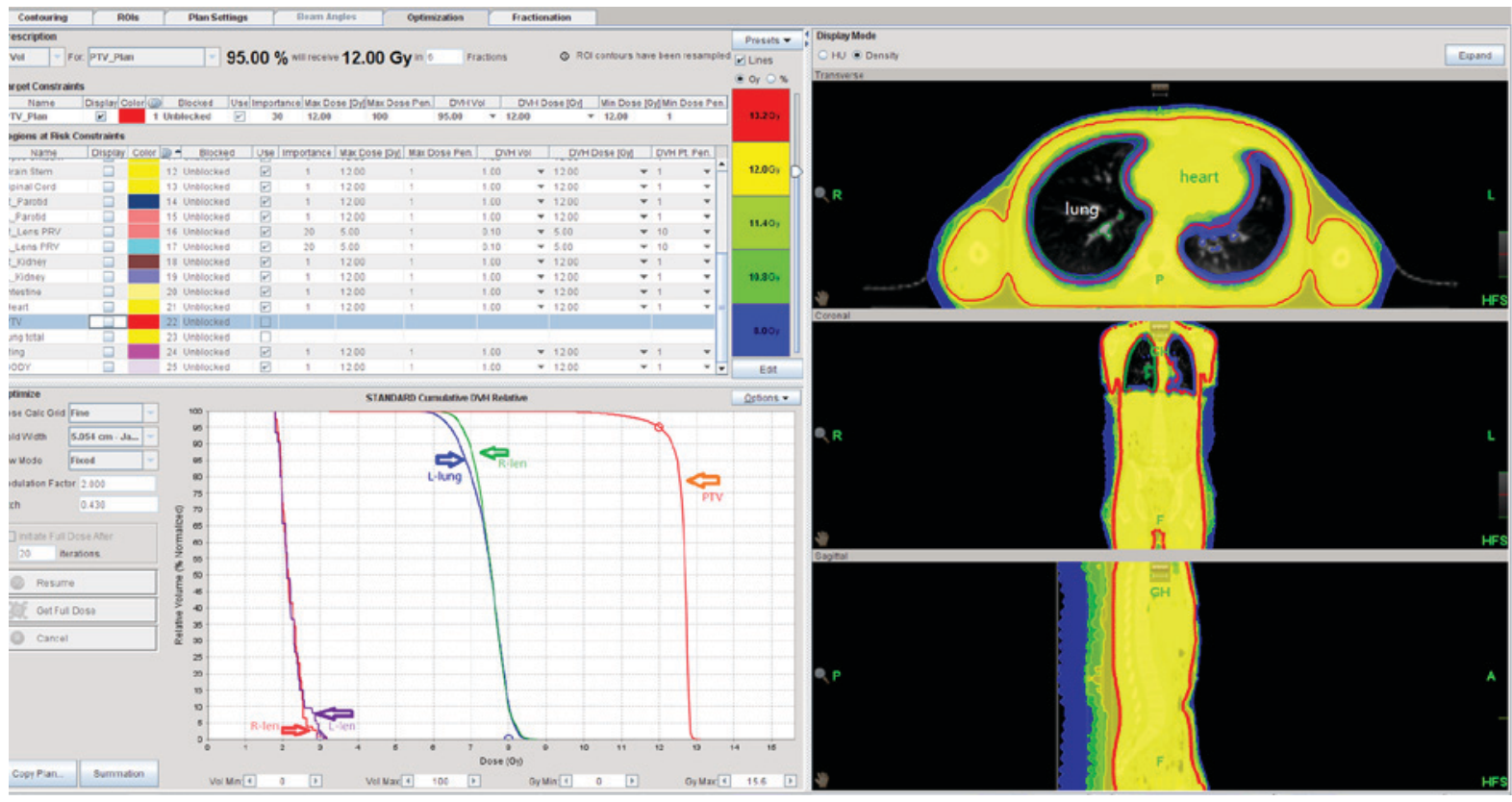

Figure 2. Dose distribution maps of patient 3 treated with TBI. The radiotherapy plan of this patient was helical tomotherapy-TBI. The red range in the map is the isodose line of $13.2 \mathrm{~Gy}$. The yellow range is the isodose line of $12.0 \mathrm{~Gy}$. The yellow-green range is the isodose line of $11.4 \mathrm{~Gy}$. The green range is the isodose line of $10.8 \mathrm{~Gy}$. The blue range is the isodose line of $8.0 \mathrm{~Gy}$. TBI, total body irradiation.

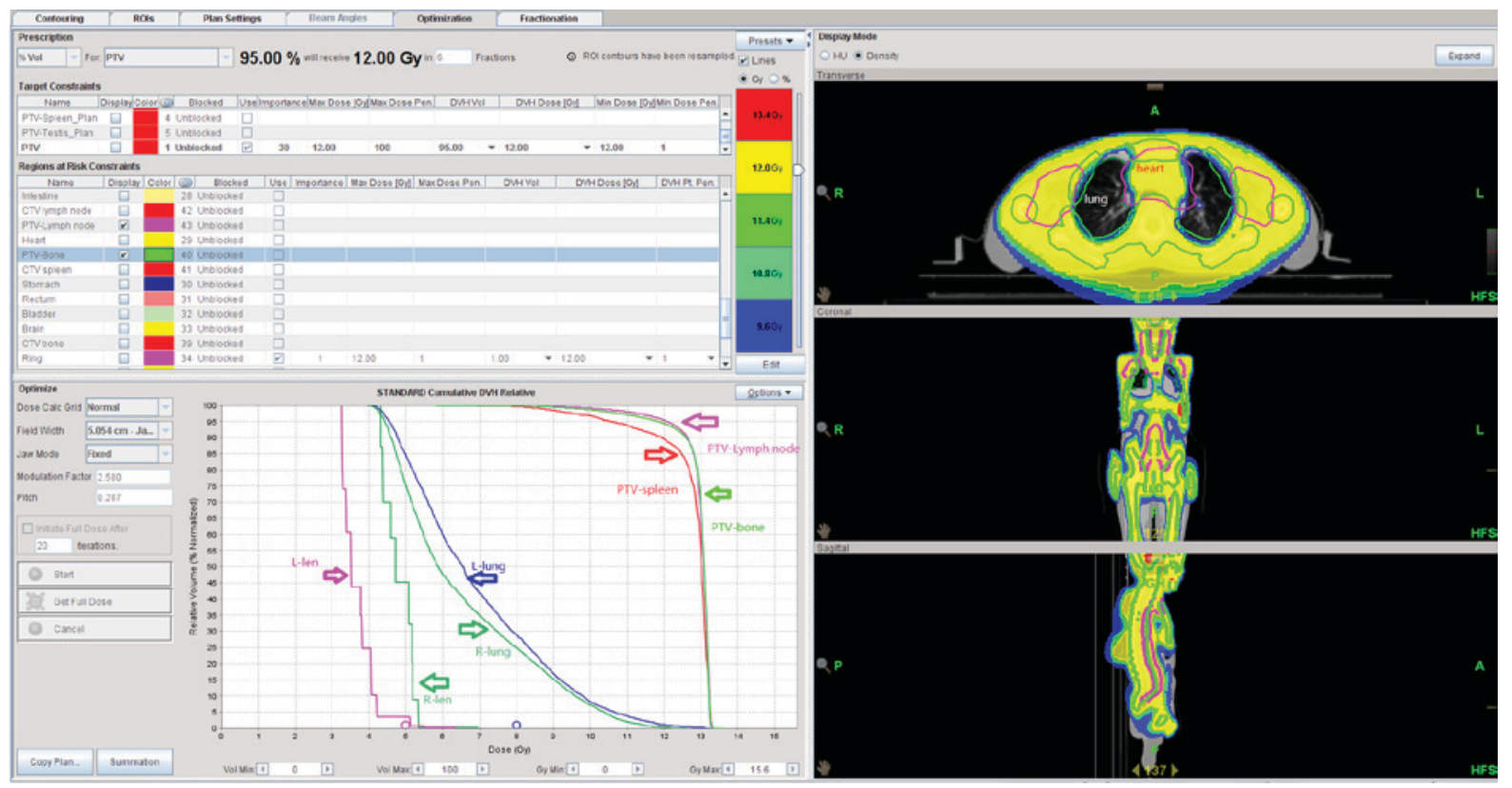

Figure 3. Dose distribution maps of patient 10 treated with TMLI. The radiotherapy plan of this patient was helical tomotherapy-TMLI. The red range is the isodose line of $13.4 \mathrm{~Gy}$. The yellow range is the isodose line of $12.0 \mathrm{~Gy}$. The green range is the isodose line of $11.4 \mathrm{~Gy}$. The light blue range is the isodose line of $10.8 \mathrm{~Gy}$. The deep blue range is the isodose line of $9.6 \mathrm{~Gy}$. TMLI, total marrow and lymphatic irradiation.

fourth treatment. Rashes improved following the radiotherapy plan and did not affect the implementation of radiotherapy plan.

According to the reactions of 18 patients following treatment, the four radiotherapy plans revealed similar untoward effects. Following symptomatic treatment, all untoward effects did not affect the implementation of radiotherapy plan.

Comparison of transplantation effects. All radiotherapy plans were completed on time in 18 patients, with a total dose of 12
Gy. All patients successfully completed transplantation. As of March 2017, the duration of follow-up was 3-8 months and the median duration of follow-up was 7 months. There were five survivors and one case of mortality (severe rejection reaction following transplantation; mortality due to severe infection) among the six patients treated with HT-TBI. A total of four patients treated with HT-TMLI survived without exhibiting a rejection response following transplantation. In addition, four patients treated with IMRT-TBI and four patients treated with IMRT-TMLI survived without rejection following 


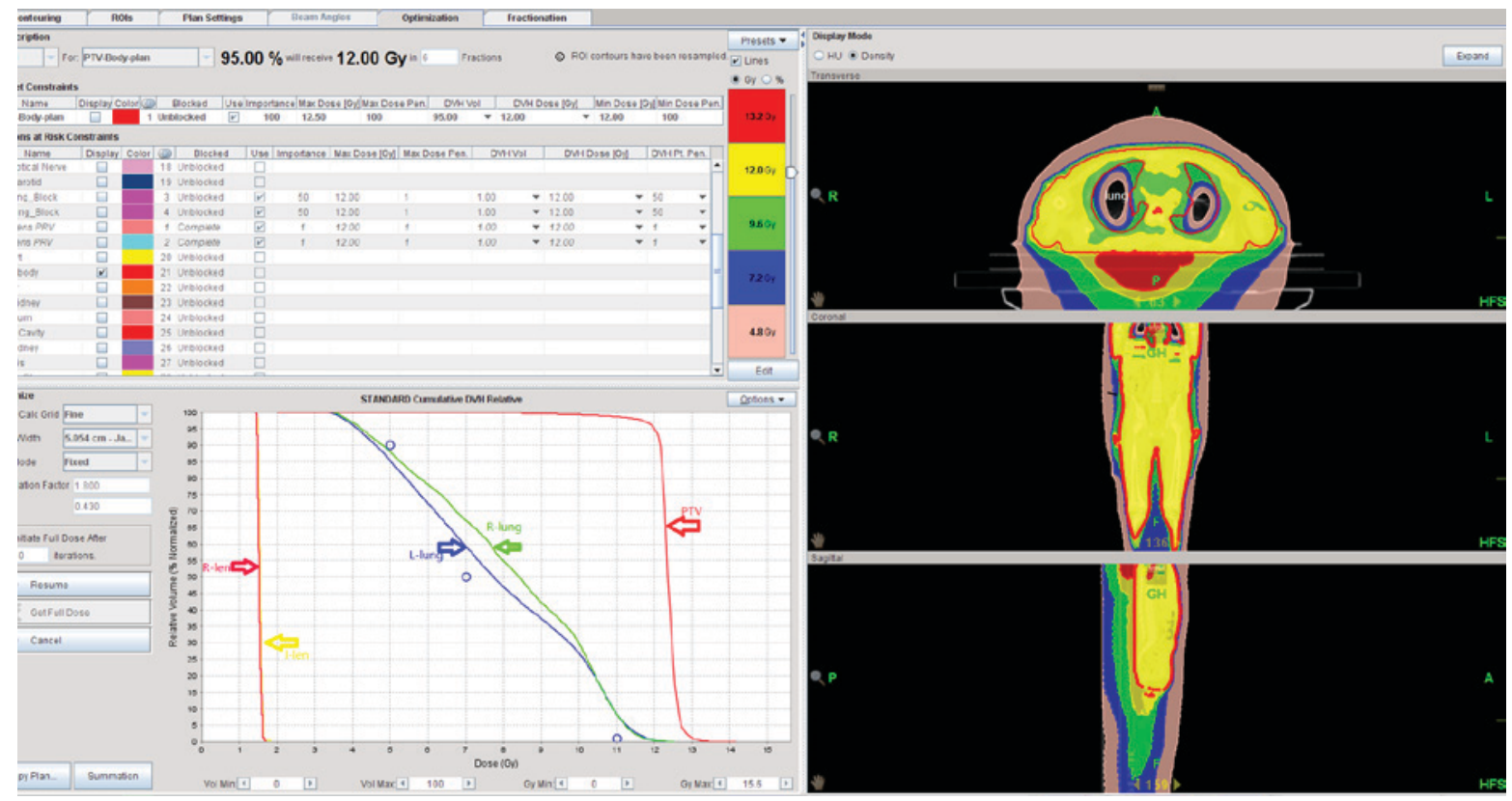

Figure 4. Dose volume histogram of patient 2 treated with helical tomotherapy-total body irradiation. The red and yellow lines on the bottom left panel represented the left and right crystalline lens; the blue and green lines in the middle represented the left and right lungs. The red line on the right of the left panel demonstrated the planning total body target volume.

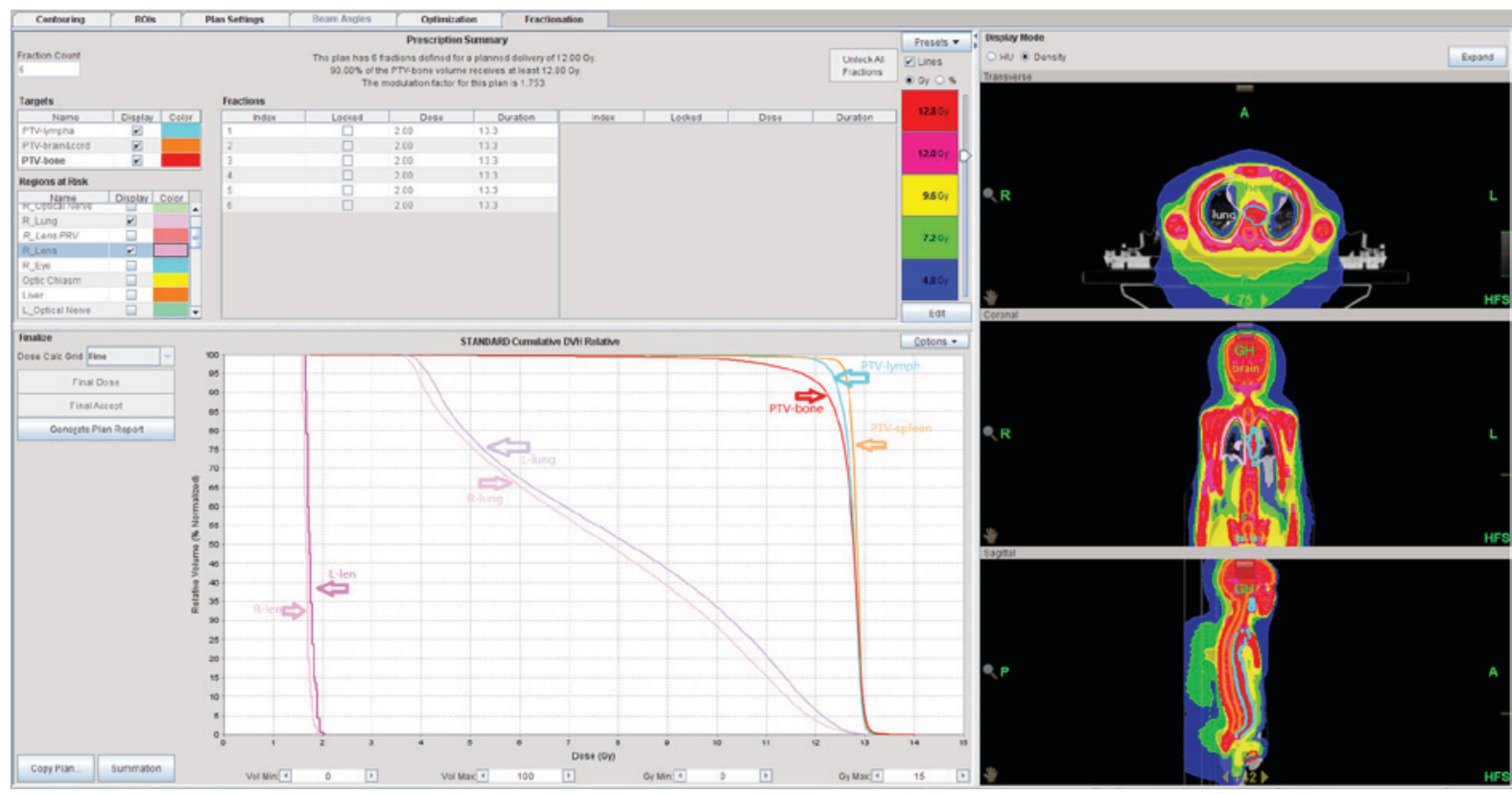

Figure 5. Dose volume histogram of patient 9 treated with helical tomotherapy-total marrow and lymphatic irradiation. The light pink and darker pink lines on the bottom left panel represented the left and right crystalline lens, respectively. The pink and purple lines in the middle are the left and right lungs, respectively. The red, light blue and orange lines on the right are PTV bone, PTV lymph node and PTV spleen, respectively. PTV, planning target volume.

transplantation. Furthermore, the remaining 17 patients were examined regularly and no severe untoward effects were observed (Figs. 2-5).

\section{Discussion}

The pathogenesis of ALL is unclear due to complicated etiology, and, therefore treatment is difficult to administer. There is a notable heterogeneity between the treatment and prognosis of ALL. The primary clinical therapy of ALL is combinational chemotherapy. Targeted therapy and hematopoietic stem cell transplantation are additional methods of treatment. Hematopoietic stem cell transplantation via high-dose chemo-radiotherapy or other immunosuppression pretreatment, removes tumor cells and abnormal clone cells in vivo of patients, and subsequent hematopoietic and immune function via the use of hematopoietic stem cells from the donor was reconstructed (33). The present clinical study demonstrated that hematopoietic stem cell transplantation may be considered as the only cure for ALL. 
TBI, as an important pretreatment method of hematopoietic stem cell transplantation $(10,11)$, concerns the success or failure of transplantation (12). Tomotherapy accelerators have been used in clinical treatment in the First Affiliated Hospital of Zhengzhou University for a number of patients against solid tumors in combination with TBI, particularly for patients with complex target volumes or who do not qualify for conventional linear accelerators therapy due to their condition. Compared with the conventional IMRT plan, the tomotherapy plan exhibits a higher conformity and increased protection to lung tissue, which may effectively reduce the incidence of radiation pneumonia (34). As of May 2016, tomotherapy accelerator in the First Affiliated Hospital of Zhengzhou University has treated numerous patients with ALL, aplastic anemia or lymphoma. A total of 18 patients with ALL in the present study were treated with HT-TBI, HT-TMLI, IMRT-TMLI and IMRT-TBI. Various radiotherapy plans were established depending on different target volumes. The results of indexes in each radiotherapy plan revealed that all plans had high conformity and homogeneity, and that doses to target volumes may meet the requirement of $12 \mathrm{~Gy}$. The doses to organs at risk were controlled below $12 \mathrm{~Gy}$; in particular, the average dose to the lung was controlled below $8 \mathrm{~Gy}$. HT plans were significantly different from IMRT plans. HT-TMLI demonstrated more advantages than the aforementioned plans. The DVH revealed that lower doses were administered to the lungs in the HT-TMLI plan. Considering the key organ of the lung and the severity of interstitial pneumonia, the reduction of dosage to the lung may be beneficial to patients. There was no difference in other organs at risk among the four plans, and all plans were able to protect the organs at risk. All patients exhibited similar untoward effects during treatment, including mild-moderate nausea, emesis, inappetence and xerostomia. These untoward effects improved following clinical symptomatic treatment, and all patients revealed tolerance to the symptomatic drugs, which did not affect the implementation of the radiotherapy plans. In the preset study, successful transplantation of the 18 patients was reported. Following transplantation, one patient succumbed to mortality due to severe infection; no severe untoward effects occurred in the remaining patients. However, the long-term effects required continuous follow-up observation.

By comparing the target volumes, clinical reactions and transplant effects among HT-TBI, HT-TMLI, IMRT-TBI and IMRT-TMLI, the clinical reactions of all plans were similar for patients with ALL. The CI of HT plans was increased compared with IMRT plans; HT-TMLI revealed higher conformity in the target volume and lower doses to organs at risk. In accordance with a previous study, the present study reported that HT-TMLI did not increase the risk of extramedullary relapse (23). Therefore, the use of HT-TMLI may be preferred over the aforementioned plans. This laid a theoretical foundation for the implementation of TBI for ALL; however, due to the relatively small number of patients and short duration of follow-up, further investigation is required to observe the long-term effects, transplantation complications and DFS to provide more accurate information for clinical treatment.

Clinical comparison of HT-TBI, HT-TMLI, IMRT-TBI and IMRT-TMLI among the 18 patients with ALL in the present study demonstrated that HT plans exhibited increased conformity compared with IMRT plans; HT-TMLI may reduce doses exposed to key organs without increasing the recurrence rate following hematopoietic stem cell transplantation, as well as attaining the requirement of conformity and dose distribution in target volume. In addition, with reference to another study (35), HT-TMLI may increase the doses for target volumes and provide improved protection for organs at risk. HT-TBI did not serve such a role; HT-TMLI did not increase the risk of extramedullary relapse. Furthermore, the four radiotherapy plans demonstrated similar clinical untoward effects and rate of transplantation success, and it may be more feasible in dosimetry to adopt HT-TMLI than the other aforementioned plans for patients with ALL. Long-term effects, complications, transplantation recurrence and long-term survival rates require further investigation with an increased number of patients to follow up, in addition to prolonged follow-up time.

\section{Acknowledgements}

Not applicable.

\section{Funding}

No funding was received.

\section{Availability of data and materials}

All data generated or analyzed during the present study are included in this published article.

\section{Authors' contributions}

YL and FK contributed to the design and concept of the present study. HL, DX, FJ and XZ acquired the data and drafted the paper. YL, FK, BW and GL analyzed and interpreted the patient data, and critically revised the paper. All authors read and approved the final manuscript.

\section{Ethics approval and consent to participate}

The present study was approved by the Ethics Committee of the First Affiliated Hospital of Zhengzhou University (Zhengzhou, China). Prior to radiotherapy, all patients and their families were notified of the requirement of treatment and possible untoward effects, and signed written informed consent was obtained.

\section{Patient consent for publication}

Not applicable.

\section{Competing interests}

The authors declare that they have no competing interests.

\section{References}

1. Ye T, Ma L and Luo S: Advances in the treatment of acute lymphoblastic leukemia. In: Proceedings of the Fifth National Anatomy and Technology Conference. Three Gorges University, Fuzhou, pp301-302, 2015. 
2. Zheng HY: Advancement of treatment of acute lymphoblastic leukemia in children. J Appl Clin Pediat 22: 167-169, 2007.

3. Pui CH, Mullighan CG, Evans WE and Relling MV: Pediatric acute lymphoblastic leukemia: Where are we going and how do we get there? Blood 120: 1165-1174, 2012

4. Hunger SP, Loh ML, Whitlock JA, Winick NJ, Carroll WL, Devidas M and Raetz EA; COG Acute Lymphoblastic Leukemia Committee: Children's Oncology Group's 2013 blueprint for research: Acute lymphoblastic leukemia. Pediatr Blood Cancer 60: 957-963, 2013.

5. Sun Q, Tang J and Huang MY: Advancement of treatment plan of acute lymphoblastic leukemia in children. Med Inform 28: 389-389, 2015

6. Hu YY and Ge FM: Advancement of treatment of acute lymphoblastic leukemia in adult. J Pract Oncol 29: 591-594, 2014

7. Linker CA, Levitt LJ, O'Donnell M, Forman SJ and Ries CA: Treatment of adult acute lymphoblastic leukemia with intensive cyclical chemotherapy: A follow-up report. Blood 78: 2814-2822, 1991.

8. Zhang QG, Xie ST, Zeng QF, Xiao Y and Liu X: Effect and toxic and side effects of TBI treating refractory lymphoma in APBSCT. Contemp Med 34: 1-3, 2014.

9. Kim I, Park S, Kim BK, Chang HM, Bang SM, Byun JH, Kim DJ, Min WS, Kim HJ and Kim CC: Allogeneic bone marrow transplantation for chronic myeloid leukemia: A retrospective study of busulfan-cytoxan versus total body irradiation-cytoxan as preparative regimen in Koreans. Clin Transplant 15: 167-172, 2001

10. Nakasone H, Fukuda T, Kanda J, Mori T, Yano S, Kobayashi T, Miyamura K, Eto T, Kanamori H, Iwato K, et al: Impact of conditioning intensity and TBI on acute GVHD after hematopoietic cell transplantation. Bone Marrow Transplant 50: 559-565, 2015.

11. Valente M, Denis J, Grenier N, Arvers P, Foucher B, Desangles F, Martigne P, Chaussard H, Drouet M, Abend M and Hérodin F: Revisiting biomarkers of total-body and partial-body exposure in a baboon model of irradiation. PLoS One 10: e0132194, 2015.

12. Ringdén O, Ruutu T, Remberger M, Nikoskelainen J, Volin L, Vindel $\varnothing v$ L, Parkkali T, Lenhoff S, Sallerfors B, Ljungman P, et al: A randomized trial comparing busulfan with total body irradiation as conditioning in allogeneic marrow transplant recipients with leukemia: A report from the Nordic Bone Marrow Transplantation Group. Blood 83: 2723-2730, 1994.

13. Blaise D, Maraninchi D, Michallet M, Reiffers J, Jouet JP, Milpied N, Devergie A, Attal M, Sotto JJ, Kuentz M, et al: Long-term follow-up of a randomized trial comparing the combination of cyclophosphamide with total body irradiation or busulfan as conditioning regimen for patients receiving HLA-identical marrow grafts for acute myeloblastic leukemia in first complete remission. Blood 97: 3669-3671, 2001.

14. Dusenbery KE, Daniels KA, McClure JS, McGlave PB Ramsay NK, Blazar BR, Neglia JP, Kersey JH and Woods WG: Randomized comparison of cyclophosphamide-total body irradiation versus busulfan-cyclophosphamide conditioning in autologous bone marrow transplantation for acute myeloid leukemia. Int J Radiat Oncol Biol Phys 31: 119-128, 1995.

15. Bunin N, Aplenc R, Kamani N, Shaw K, Cnaan A and Simms S: Randomized trial of busulfan vs total body irradiation containing conditioning regimens for children with acute lymphoblastic leukemia: A Pediatric Blood and Marrow Transplant Consortium study. Bone Marrow Transplant 32: 543-548, 2003

16. Farese AM, Brown CR, Smith CP, Gibbs AM, Katz BP, Johnson CS, Prado KL and MacVittie TJ: The ability of filgrastim to mitigate mortality following LD50/60 total-body irradiation is administration time-dependent. Health Phys 106: 39-47, 2014.

17. Burnett AF, Biju PG, Lui H and Hauer-Jensen M: Oral interleukin 11 as a countermeasure to lethal total-body irradiation in a murine model. Radiat Res 180: 595-602, 2013.

18. Nunamaker EA, Anderson RJ, Artwohl JE, Lyubimov AV and Fortman JD: Predictive observation-based endpoint criteria for mice receiving total body irradiation. Comp Med 63: 313-322, 2013

19. Linsenmeier C, Thoennessen D, Negretti L, Bourquin JP, Streller T, Lütolf UM and Oertel S: Total body irradiation (TBI) in pediatric patients. A single-center experience after 30 years of low-dose rate irradiation. Strahlenther Onkol 186: 614-620, 2010

20. Bhatia S, Francisco L, Carter A, Sun CL, Baker KS, Gurney JG, McGlave PB, Nademanee A, O'Donnell M, Ramsay NK, et al: Late mortality after allogeneic hematopoietic cell transplantation and functional status of long-term survivors: Report from the Bone Marrow Transplant Survivor Study. Blood 110: 3784-3792, 2007.
21. Bhatia S, Robison LL, Francisco L, Carter A, Liu Y, Grant M, Baker KS, Fung H, Gurney JG, McGlave PB, et al: Late mortality in survivors of autologous hematopoietic-cell transplantation: Report from the Bone Marrow Transplant Survivor Study. Blood 105: 4215-4222, 2005.

22. Appelbaum FR, Badger CC, Bernstein ID, Buckner CD, Deeg HJ, Eary JF, Matthews DC, Press OW, Storb R and Thomas ED: Is there a better way to deliver total body irradiation? Bone Marrow Transplant 10 (Suppl 1): S77-S81, 1992.

23. Kim JH, Stein A, Tsai N, Schultheiss TE, Palmer J, Liu A, Rosenthal J, Forman SJ and Wong JY: Extramedullary relapse following total marrow and lymphoid irradiation in patients undergoing allogeneic hematopoietic cell transplantation. Int J Radiat Oncol Biol Phys 89: 75-81, 2014.

24. Lee KH, Lee JH, Choi SJ, Lee JH, Kim S, Seol M, Lee YS, Kim WK, Seo EJ, Park CJ, et al: Bone marrow vs extramedullary relapse of acute leukemia after allogeneic hematopoietic cell transplantation: Risk factors and clinical course. Bone Marrow Transplant 32: 835-842, 2003.

25. Chong G, Byrnes G, Szer J and Grigg A: Extramedullary relapse after allogeneic bone marrow transplantation for haematological malignancy. Bone Marrow Transplant 26: 1011-1015, 2000

26. Solh M, DeFor TE, Weisdorf DJ and Kaufman DS: Extramedullary relapse of acute myelogenous leukemia after allogeneic hematopoietic stem cell transplantation: Better prognosis than systemic relapse. Biol Blood Marrow Transplant 18: $106-112,2012$

27. Mortimer J, Blinder MA, Schulman S, Appelbaum FR, Buckner CD, Clift RA, Sanders JE, Storb R and Thomas ED: Relapse of acute leukemia after marrow transplantation: Natural history and results of subsequent therapy. J Clin Oncol 7: 50-57, 1989.

28. Wong JY, Forman S, Somlo G, Rosenthal J, Liu A, Schultheiss T, Radany E, Palmer J and Stein A: Dose escalation of total marrow irradiation with concurrent chemotherapy in patients with advanced acute leukemia undergoing allogeneic hematopoietic cell transplantation. Int J Radiat Oncol Biol Phys 85: 148-156, 2013.

29. Rosenthal J, Wong J, Stein A, Qian D, Hitt D, Naeem H, Dagis A, Thomas SH and Forman S: Phase 1/2 trial of total marrow and lymph node irradiation to augment reduced-intensity transplantation for advanced hematologic malignancies. Blood 117: 309-315, 2011.

30. Karnofsky DA and Burchenal JH: The clinical evaluation of chemotherapeutic agents in cancer. In: Evaluation of Chemotherapeutic Agents. Macleod CM (ed). Columbia University Press, New York, NY, p191, 1949.

31. Trotti A, Byhardt R, Stetz J, Gwede C, Corn B, Fu K, Gunderson L, McCormick B, Morrisintegral M, Rich T, et al: Common toxicity criteria: Version 2.0. an improved reference for grading the acute effects of cancer treatment: Impact on radiotherapy. Int J Radiat Oncol Biol Phys 47: 13-47, 2000.

32. U.S. Department of Health and Human Services, The National Institutes of Health and National Cancer Institute: Common Terminology Criteria for Adverse Events (CTCAE) Version 4.0.3. https://evs.nci.nih.gov/ftp1/CTCAE/CTCAE_4.03/ CTCAE 4.03_2010-06-14_QuickReference_5x7.pdf. Accessed June $14,2010$.

33. Storek J, Geddes M, Khan F, Huard B, Helg C, Chalandon Y, Passweg J and Roosnek E: Reconstitution of the immune system after hematopoietic stem cell transplantation in humans. Semin Immunopathol 30: 425-437, 2008.

34. Moon SH, Shin KH, Kim TH, Yoon M, Park S, Lee DH, Kim JW, Kim DW, Park SY and Cho KH: Dosimetric comparison of four different external beam partial breast irradiation techniques: Three-dimensional conformal radiotherapy, intensity-modulated radiotherapy, helical tomotherapy, and proton beam therapy. Radiother Oncol 90: 66-73, 2009.

35. Schultheiss TE, Wong J, Liu A, Olivera G and Somlo G Image-guided total marrow and total lymphatic irradiation using helical tomotherapy. Int J Radiat Oncol Biol Phys 67: 1259-1267, 2007.

This work is licensed under a Creative Commons Attribution-NonCommercial-NoDerivatives 4.0 International (CC BY-NC-ND 4.0) License. 